\title{
Vortices in Bose-Einstein condensates confined in a multiply connected Laguerre-Gaussian optical trap
}

\author{
J. Tempere and J. T. Devreese \\ Departement Natuurkunde, Universiteit Antwerpen UIA, Universiteitsplein 1, B-2610 Antwerpen, Belgium \\ E. R. I. Abraham \\ Department of Physics and Astronomy, University of Oklahoma, 440 W. Brooks, Norman, Oklahoma 73019
}

(Received 29 February 2000; revised manuscript received 21 December 2000; published 5 July 2001)

\begin{abstract}
The quantized vortex state is investigated in a Bose-Einstein condensate, confined in a multiply connected geometry formed by a Laguerre-Gaussian optical trap. Solving the Gross-Pitaevskii equation variationally, we show that the criterion for vortex stability is that the interatomic interaction strength must exceed a critical interaction strength. The time evolution of a freely expanding Laguerre-Gaussian condensate with a vortex is calculated and used to derive the interference pattern of such a condensate overlapping with a parabolically trapped condensate.
\end{abstract}

DOI: 10.1103/PhysRevA.64.023603

PACS number(s): 03.75.Fi, 05.30.Jp, 32.80.Pj

\section{INTRODUCTION}

Superfluidity, and its characteristic manifestation as a state with quantized circulation (a vortex), is intimately connected with the phenomenon of Bose-Einstein condensation, yet the precise relation between superfluid persistent currents and Bose-Einstein condensation (BEC) is only beginning to be elucidated [1]. Before 1995, the link between superfluidity and BEC was almost exclusively studied in the context of liquid helium $\left({ }^{4} \mathrm{He}\right.$ and $\left.{ }^{3} \mathrm{He}\right)$ [2], where the study of the relation between superfluidity and BEC is complicated by the strong interatomic interactions between the atoms in the liquid. In 1995, Bose-Einstein condensation was realized in magnetically trapped clouds of alkali-metal atoms [3]. In these novel condensates, the bosonic atoms are weakly interacting (contrary to the case of liquid helium), and as such these systems have the potential to shed new light on superfluidity. Soon after the initial creation of alkali-metal gas condensates, several experimental groups set out to create a vortex - a quantum of superfluid circulation - in this novel system [4].

The initial attempts to create a vortex in a condensate by stirring the trapped condensate with a blue detuned laser beam [4] were unsuccessful, even though early theoretical work by Dalfovo et al. indicated that persistent superfluid currents can indeed manifest themselves in Bose-Einstein condensates as vortices [5]. Subsequent analysis [6] showed that vortices are unstable in the simply connected, notstirred, spin-polarized condensates formed in the original experiments [3].

This can be understood as follows. Along the vortex line, the order parameter of the Bose-Einstein condensate has to vanish. Phrased metaphorically, a "hole" has to be "drilled" in the condensate along the vortex line. In the magnetic trap, the modulus square of the order parameter of a condensate without a vortex is largest in the center of the trap. As a consequence, a vortex line through the center of the trap will perturb the order parameter more than a vortex line at the edge of the condensate (since, using our metaphor again, it will cost more energy to "drill" through the center of the condensate than through its edge). It will be energetically favorable for the vortex line to be at the edge of the condensate. In the presence of dissipation, this will cause the vortex line to migrate to the edge of the condensate so that the vortex condensate will decay into a nonvortex state. This argument, sketched here with some roughness, has been worked out with precision by Fetter and co-workers in [7].

Several schemes have been proposed, both theoretically and experimentally, to stabilize vortices: rotating the trapping potential (analogous to rotating a bucket containing ${ }^{4} \mathrm{He}$ ) [6] or stirring the condensate with an off-resonance laser [8], raising the temperature (to "pin" the vortex in the potential created by the noncondensate fraction at the center of the vortex) [9], phase-imprinting methods [10], and various other techniques [11]. Recently, vortices were created experimentally, both with a "rotating bucket" experiment [12] and with the use of a two-component condensate [13]. In the latter experiment, one of the components of the spinor condensate "pins" the vortex present in the other component and a Ramsey-type interference between the two components is used to detect the vortex. The long lifetimes of the twocomponent condensates in Ref. [13] are due to similar singlet and triplet scattering lengths of rubidium, resulting in an anomalously low inelastic loss rate [14].

Nevertheless, stable vortices have not yet been realized in a spin-polarized condensate in nonrotating traps, and new methods of stabilization and detection must be developed. A promising scheme, based on an analysis similar to that of Fetter [7], is the use of multiply connected condensates. A candidate trap to create a multiply connected condensate consists of a red-detuned laser beam in a Laguerre-Gauss mode $[15,16]$, which we discuss in Sec. II. If the condensate order parameter has a toroidal geometry, a vortex line threaded through the cylindrical symmetry axis of the torus will not perturb the condensate order parameter strongly, and moreover create a metastability barrier for vortex decay. One of the goals of this paper is to verify this statement about vortex stability (in Sec. III). In a condensate with a toroidal order parameter, the vortex line can no longer be detected as a line along which the density of Bose-Einstein condensed 


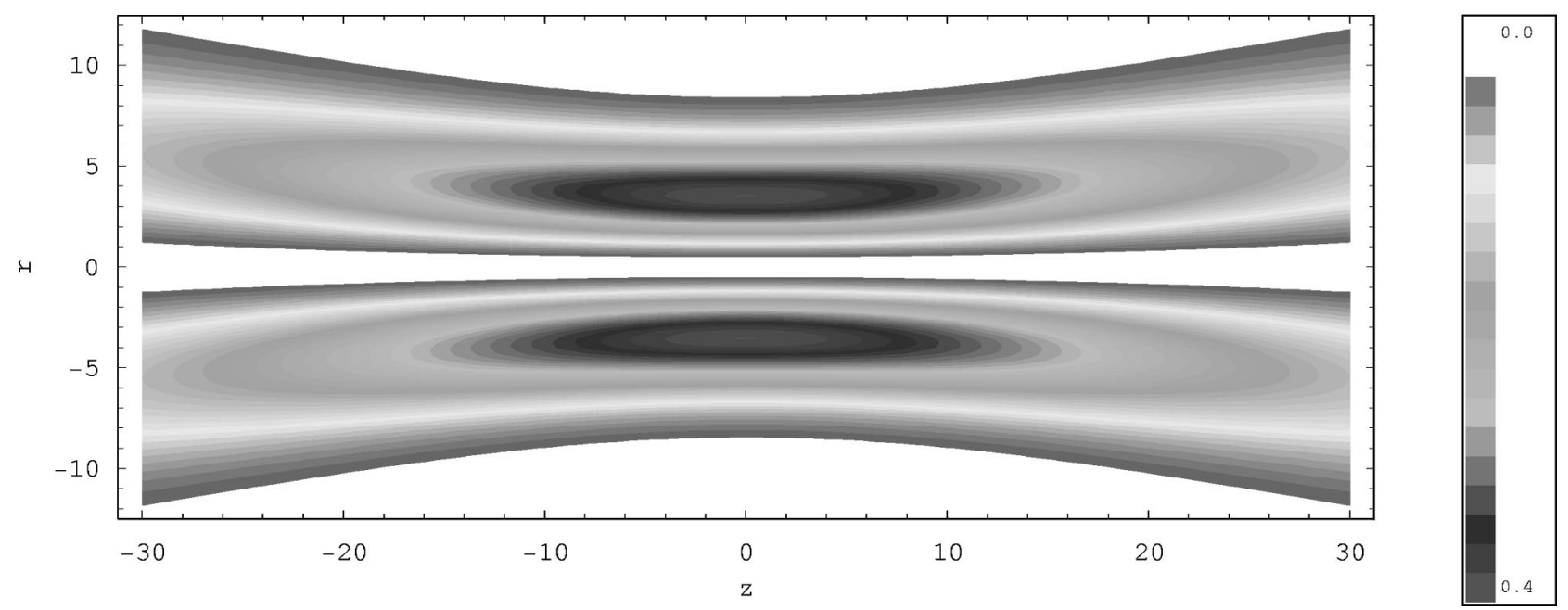

FIG. 1. The Laguerre-Gauss (LG) trap is an optical dipole trap consisting of a red-detuned laser beam. A typical intensity profile of the laser beam in the LG mode $\{0,1\}$ is shown in this figure, in a cross section through the axis of propagation of the beam (the $z$ axis). The atoms will feel the optical dipole force attracting them to the (toroidal) region of highest intensity of the laser beam.

atoms vanishes. To detect vorticity, we propose (in Sec. IV) a method based on interference, similar to that proposed in Refs. $[17,18]$ for simply connected condensates.

\section{BOSE-EINSTEIN CONDENSATION IN LAGUERRE-GAUSS TRAPS}

\section{A. Trapping geometry}

Toroidal confinement for ultracold atoms can be obtained by an optical dipole trap [19], which consists of a laser beam in a Laguerre-Gaussian mode. The Laguerre-Gaussian mode $\{n, m\}$ is characterized by an intensity profile given by [20]

$$
I_{n, l}(r, z) \propto \frac{\left(2 r^{2} / W_{0}^{2}\right)^{l}}{1+\left(2 z / k W_{0}^{2}\right)^{2}} L_{n, l}^{2}\left(2 r^{2} / W_{0}^{2}\right) \exp \left\{-\frac{2 r^{2}}{W_{0}^{2}}\right\},
$$

where $r$ is the radial distance from the center of the beam, $z$ is the position along the propagation direction of the beam, $W_{0}$ (referred to as the "waist parameter") is a parameter controlling the minimal width of the beam, $k$ is the wave number of the laser, and $L_{n, l}$ is the Laguerre polynomial of order $\{n, l\}$. Such laser beams have modes with $l>0$ that show a node in the center, and trap the atoms in a cylindrical shell around the axis of propagation of the beam. The intensity profile of the laser beam in the Laguerre-Gaussian mode is illustrated in Fig. 1. Laguerre-Gaussian (LG) laser beams have already been used to successfully trap atoms [16], and subsequent theoretical work has shown that toroidal traps formed by a red-detuned LG beam can be loaded from initial conditions similar to those of conventional magnetic traps [21]. Once the trap has been loaded, one possibility to create a vortex in the trapped gas would be by a phase imprinting method, which already successfully resulted in the creation of solitons in condensates [22,23], but might be difficult to use in practice for vortices [23]. Alternatively, a rotating perturbing potential can be used to stir the condensate and set up persistent flow in the toroidal geometry [24].
In this paper, we investigate the properties of the vortex Bose-Einstein condensate, optically trapped by a laser beam in a Laguerre-Gaussian propagation mode $\{n, l\}=\{0,1\}$. Along the $z$ axis (the direction of propagation of the laser beam), an additional magnetic trap [15] results in a parabolic $z$-axis confinement with frequency $\Omega$, which prevents the atoms from escaping along the direction of propagation of the laser beam. This "plugging" of the optical trap was achieved in [16] by using blue-detuned "plugging beams." The condensate in the Laguerre-Gauss geometry [15] will be called the "Laguerre-Gaussian condensate" (LG condensate), in contrast with the condensate in a parabolic confinement.

The intensity profile of such a Laguerre-Gauss beam in the $z y$ plane (where $z$ is the axis of propagation of the laser beam), given by Eq. (1), is shown in Fig. 1. In the remainder of this paper, we use units so that $\hbar=m=\Omega=1$ (where $m$ is the mass of the atoms). In these units, the laser beam parameters for Fig. 1 are chosen as follows: $k=2, W_{0}=5$. Figure 2 illustrates a surface of constant intensity of the laser beam, a hollow cylindrical shell. This will also be the shape of the cloud of trapped atoms [21]. The extension of the cloud

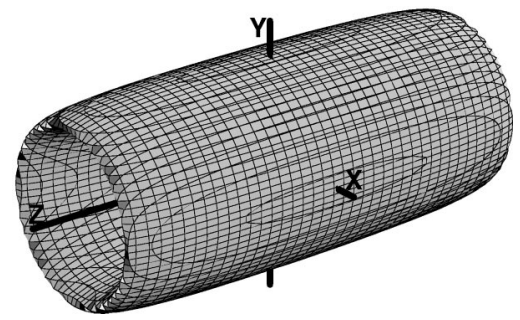

FIG. 2. A typical surface of constant intensity [expression (1)] of the laser beam in the Laguerre-Gauss (LG) mode $\{0,1\}$ is shown. The atoms in the red-detuned LG beam will be attracted to a toroidal or cylindrical region such as that within the surface of the figure. An additional parabolic magnetic confinement potential can be added in the $z$ direction $[15,16]$. 
along the $z$ axis can be tuned by selecting the $\Omega$ frequency of the magnetic trap along the $z$ axis.

\section{B. Mean-field approach}

Confined Bose-Einstein condensates are well described by a mean-field theory where the properties of the condensate are derived from a complex function $\Psi$. This function (the order parameter) is interpreted as a macroscopic wave function and obeys the Gross-Pitaevskii equation [25]

$$
-\frac{\hbar^{2}}{2 m} \Delta \Psi+V_{\text {conf }}(\rho, z) \Psi+U_{0}|\Psi|^{2} \Psi=E \Psi .
$$

The term nonlinear in $\Psi$ in Eq. (2) arises from the interparticle interaction potential, which is treated as a contact potential with scattering length $a_{\text {scat }}$ so that $U_{0}=4 \pi \hbar^{2} a_{\text {scat }} / m$, where $m$ is the mass of an atom. The optical confinement is generated by a red-detuned Laguerre-Gaussian laser beam as discussed in the preceding section. In addition to this, a harmonic confinement (characterized by a frequency $\Omega$ ) is present that confines the atoms along the $z$ direction to a region $z<k W_{0}^{2}$ [15]. The potential energy corresponding to this geometry is given, in cylindrical coordinates $\{\rho, \theta, z\}$, by

$$
V_{\text {conf }}(\rho, \theta, z)=-A_{0} \frac{2 \rho^{2}}{W_{0}^{2}} \exp \left\{-2 \rho^{2} / W_{0}^{2}\right\}+\frac{m \Omega^{2}}{2} z^{2}
$$

Kuga et al. [16] report that for a $\{0,3\}$ LG beam with a laser power of $600 \mathrm{~mW}$, the depth of the trap is enough to trap atoms that have been precooled to $10 \mu \mathrm{K}$. In the present treatment, the Gross-Pitaevskii equation (2) will be solved variationally [26]. Consider for this purpose the following two trial functions, expressed in cylindrical coordinates $\{\rho, \theta, z\}$ :

$$
\begin{gathered}
\psi(\rho, \theta, z)=\mathcal{N}_{0} \exp \left\{-\sigma_{0}\left(\rho-\rho_{0}\right)^{2} / 2-\zeta_{0} z^{2} / 2\right\} \\
\psi_{v}(\rho, \theta, z)=\mathcal{N}_{1} \rho \exp \left\{-\sigma_{1}\left(\rho-\rho_{1}\right)^{2} / 2-\zeta_{1} z^{2} / 2\right\} e^{i \theta} .
\end{gathered}
$$

The variational parameters in the trial functions are $\sigma_{0}, \sigma_{1}$, controlling the width of the functions in the radial direction; $\zeta_{0}, \zeta_{1}$, controlling the widths in the $z$ direction; and $\rho_{0}, \rho_{1}$, controlling the radial displacement of the maximum of the function away from the $z$ axis. $\mathcal{N}_{0}$ and $\mathcal{N}_{1}$ are normalization constants, which are determined by demanding that the norm of the trial function equals the number of particles in the condensate.

The circulation around a closed loop in the trapped, interacting Bose gas is defined by $\kappa=(\hbar / m) \oint \nabla S$, where $S$ represents the phase of the order parameter solving the GrossPitaevskii equation (2). The single-valuedness of the order parameter ensures that the circulation in the trapped Bose gas is quantized: $\kappa=n h / m$ with $n=0,1,2, \ldots$. A vortex is present whenever $n>0$. The trial function $\psi(\rho, \theta, z)$ has a constant phase and hence there is no circulation, no vortex. On the other hand, the phase of $\psi_{v}(\rho, \theta, z)$ changes by $2 \pi$ along any closed loop encircling the $z$ axis. Thus $\psi_{v}$ is a trial function for the order parameter of a condensate with one

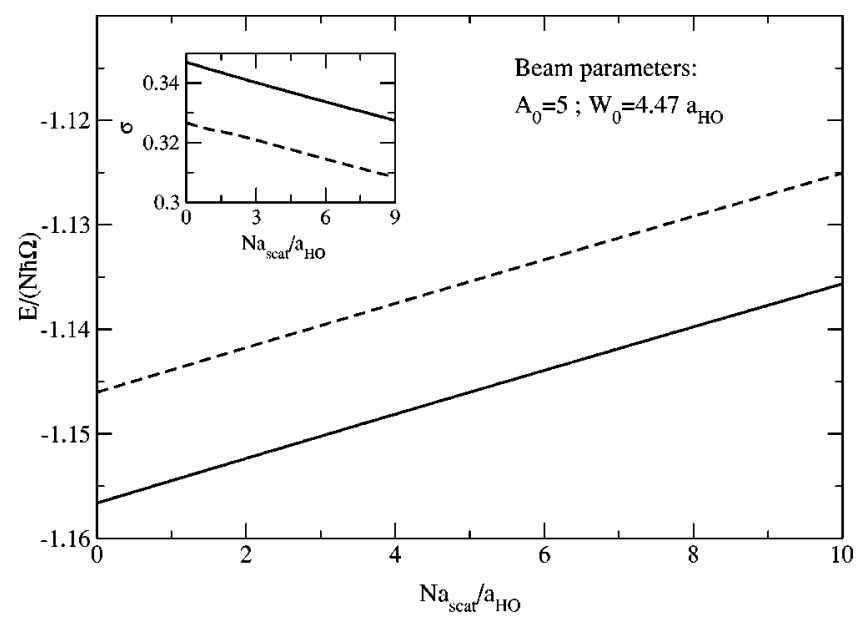

FIG. 3. The variational result for the energy of the condensate in the Laguerre-Gaussian optical trap is shown as a function of the interaction strength. The full curve shows the energy of the condensate without a vortex, the dashed curve shows the energy with a vortex. The inset depicts the results for the variation parameters $\sigma_{0}, \sigma_{1}$ in the trial function for the condensate with vortex (dashed line) and without (full line), as a function of interaction strength. The trapping (beam) parameters were chosen as follows: $A_{0}$ $=5 \hbar \Omega, W_{0}^{2}=20 a_{\mathrm{HO}}^{2}$. In all graphs of this figure, energies are expressed in units $\hbar \Omega$ and lengths in units $a_{\mathrm{HO}}=\sqrt{\hbar /(m \Omega)}$.

quantum of vorticity: its circulation equals $\kappa=h / m$. The trial function $\psi_{v}$ must have a node along the $z$ axis [the factor $\rho$ in expression (5)]: if this node were not present, the variational kinetic energy would diverge along the $z$ axis.

We have used $\psi$ and $\psi_{v}$ as variational trial functions to solve the Gross-Pitaevskii equation for a condensate without and with a vortex, respectively. The resulting variational energy is shown in Fig. 3 as a function of $N a_{\text {scat }} / a_{\mathrm{HO}}$, where $N$ is the number of atoms and $a_{\mathrm{HO}}=\sqrt{\hbar /(m \Omega)}$. The quantity $N a_{\text {scat }} / a_{\mathrm{HO}}$ is a dimensionless measure of the strength of the interaction: upon increasing $N a_{\text {scat }} / a_{\mathrm{HO}}>0$, the interatomic interaction becomes more repulsive. The energy of the LG condensate with a vortex is larger than the energy of the LG condensate without a vortex for all interaction strengths investigated. The absolute difference in total energy is smallest for the noninteracting gas and increases monotonically as $N a_{\text {scat }} / a_{\mathrm{HO}}$ increases. This means that for all investigated interaction strengths, the vortex LG condensate is either metastable or unstable. In inset (a) of Fig. 3, the optimal value of the variational parameters $\sigma$ is shown for both trial functions as a function of the interaction strength. Both the variational trial function of the LG condensate with a vortex and without a vortex are broadened under the influence of the repulsive interactions.

\section{VORTEX METASTABILITY BARRIERS}

A Laguerre-Gaussian condensate with a vortex can be metastable if there exists an energy barrier separating this state from a state without a vortex. In this section, we estimate the height of the energy barrier both by the method proposed by Benakli et al. [27] for two-dimensional (2D) 
traps and by the method proposed by Fetter and co-workers [7].

\section{A. Hydrodynamic and microscopic instability}

The hydrodynamic and microscopic instabilities studied by Fetter and co-workers [7] involve the displacement of the vortex core relative to the center of the trap. As discussed in the Introduction, for a parabolically trapped condensate it is energetically favorable for the distance between the vortex core and the center of the trap to increase. Thus, if dissipation is present, the vortex condensate in the parabolic trap can decay into a nonvortex condensate through a migration of the vortex to the edge of the cloud. This effect is difficult to observe experimentally, since the vortex core can tilt and the image contrast between the vortex core and the cloud of atoms is reduced [28].

To estimate the height of the energy barrier separating the vortex condensate from the condensate without a vortex in our present case, we introduce a third variational function to represent the order parameter of a vortex LG condensate where the vortex core is displaced a given distance $R$ along the $x$ axis, away from the cylindrical symmetry axis (the $z$ axis) of the Laguerre-Gauss trapping beam:

$$
\begin{aligned}
\psi_{v}(R ; \rho, \theta, z)= & \mathcal{N}_{2} r(\rho, \theta) \exp \left\{-\sigma_{R}\left(\rho-\rho_{R}\right)^{2} / 2\right. \\
& \left.-\zeta_{R} z^{2} / 2\right\} e^{i \Theta(\rho, \theta)} .
\end{aligned}
$$

The function $r(\rho, \theta)$ gives the distance between the point $\{\rho, \theta, 0\}$ and the vortex core at $\{R, 0,0\}$ :

$$
r(\rho, \theta)=\sqrt{(\rho \cos \theta-R)^{2}+\rho^{2} \sin ^{2} \theta},
$$

and the function $\Theta(\rho, \theta)$ gives the angle between the $\theta=0$ line (the $x$ axis) and the line connecting the vortex core at $\{R, 0,0\}$ with the point $\{\rho, \theta, 0\}$ :

$$
\Theta(\rho, \theta)=\arctan \left(\frac{\rho \sin \theta}{\rho \cos \theta-R}\right) .
$$

The parameters $\left\{\sigma_{R}, \rho_{R}, \zeta_{R}\right\}$ are determined variationally for every studied distance $R$ between the vortex core and the center of the trapping geometry (the axis of propagation of the $L G$ beam), and $\mathcal{N}_{2}$ is a normalization constant. The limit $R \rightarrow 0$ retrieves the results for the vortex condensate studied earlier. In the limit $R \rightarrow \infty$, the result tends to the result for the condensate without a vortex. Using the variational approach, we calculate the energy $E(R)$ of the displaced vortex state for any intermediate $R$. If the energy $E(R)$ decreases monotonously with increasing $R$, the vortex is unstable; in the presence of dissipation the vortex condensate will decay. If there exists a maximum energy $E\left(R_{\max }\right)$ for an $R_{\max }$ different from zero, there is an energy barrier $E_{\text {barrier }}$ $=E\left(R_{\max }\right)-E(R=0)$ that will hinder the vortex decay.

In Fig. 4, the energy barrier $E_{\text {barrier }}$ is shown as a function of $N a_{\text {scat }} / a_{\mathrm{HO}}$, the dimensionless measure of the interaction strength used in the preceding section. The calculations were performed for a configuration so that $A_{0}=5 \hbar \Omega, W_{0}^{2}$ $=20 a_{\mathrm{HO}}^{2}$. We found that a metastability barrier exists

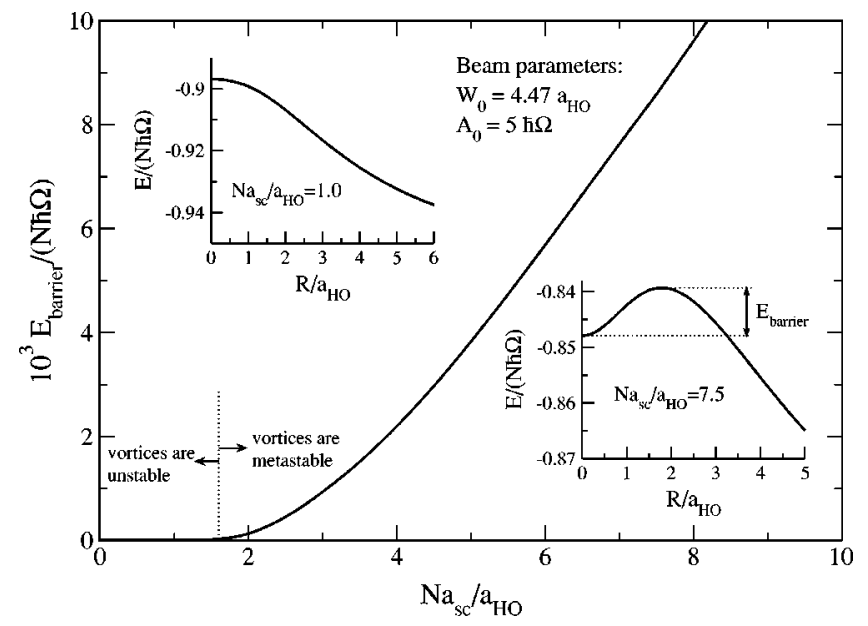

FIG. 4. The energy barrier for removing a vortex from a condensate in a Laguerre-Gaussian optical trap is depicted as a function of interaction strength. This metastability barrier $E_{\text {barrier }}$ was calculated using the formalism of Fetter and co-workers [7]. In this formalism, $E_{\text {barrier }}$ is found by deriving the energy $E(R)$ as a function of the distance $R$ between the vortex core and the center of the trapping potential, here the axis of propagation of the laser beam. This is illustrated in the insets. For interaction strengths lower than a critical value $N a_{\text {scat }} / a_{\mathrm{HO}}<1.6 \pm 0.1$, the barrier vanishes and vortices in Laguerre-Gaussian condensates are unstable with respect to the nonvortex state. For interaction strengths above this critical value, a metastability barrier exists.

$\left(E_{\text {barrier }}>0\right)$ for $N a_{\text {scat }} / a_{\mathrm{HO}}>1.6 \pm 0.1$. This means that vortices are metastable only if the effective interaction strength (controlled by the scattering length, the number of atoms, and the confinement strength) is large enough: the metastability is induced by interactions. Upon further increasing the interaction strength above the threshold value, the metastability barrier increases. The variational energy $E(R)$ is shown as a function of $R$ in the insets: once for an interaction strength such that the vortex is not stable and once for a situation in which the vortex is metastable. A reasonable estimate of the experimental parameters required to realize this trapping geometry gives $a_{\mathrm{HO}}=4 \mu \mathrm{m}$ and $\Omega=50 \mathrm{~Hz}$. This implies that for ${ }^{87} \mathrm{Rb}$, the critical number of trapped atoms necessary to make vortices metastable is of the order of $10^{3}$. Since one can reasonably expect to trap more than $10^{5}$ atoms in the trap, vortices created in a Bose gas in the confinement potential mentioned above will be well into the metastable regime $\left(N a_{\text {scat }} / a_{\mathrm{HO}} \approx 625\right.$ for $\left.N=500000\right)$.

\section{B. Uniform transition to a nonvortex state}

The other method that we used to study the metastability of the vortices in Laguerre-Gauss condensates was proposed by Benakli et al. [27] for 2D traps with an axial hole, punctured by an off-resonance laser beam. In this method, a trial solution for the Gross-Pitaevskii equation is constructed as a superposition between the vortex state and the state without a vortex: $\Psi(\rho, \theta, z)=C_{0} \psi(\rho, \theta, z)+C_{1} \psi_{v}(\rho, \theta, z)$, where $\left|C_{0}\right|^{2}+\left|C_{1}\right|^{2}=1$. Adapting the coefficients $C_{0}$ and $C_{1}$ of this superposition, the function changes from that of a LG con- 


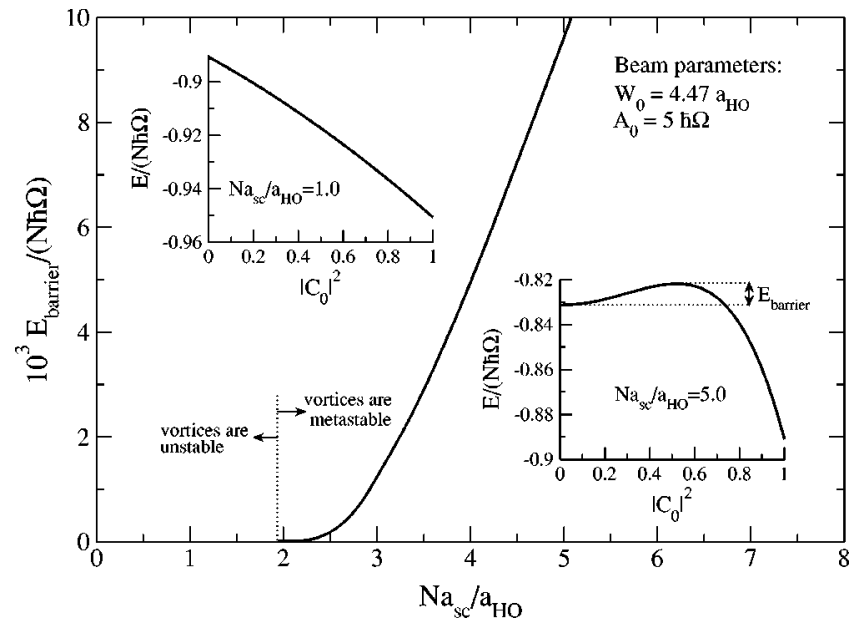

FIG. 5. The energy barrier for a uniform transition from a vortex condensate to a condensate without a vortex, in a LaguerreGaussian optical trap, is depicted as a function of interaction strength. The energy barrier for this mechanism was calculated by the method of Benakli et al. [27]. In the inset, the energy per particle of the variational function $\Psi=C_{0} \psi+C_{1} \psi_{v}$ is given as a function of $\left|C_{0}\right|^{2}$ for two different interaction strengths [one below (a) and one above (b)], the threshold for metastability of vortices, and the relation to the energy barrier for a uniform transition is shown.

densate with a vortex to that without a vortex. If the energy of the intermediate states is found to be higher than the energy of the vortex state, this constitutes an energy barrier against a uniform transition from the vortex state to the state without a vortex, making the former metastable.

Using the variationally optimized trial functions for the order parameter of a LG condensate with a vortex and without a vortex, we found with the method of Benakli et al. that such a metastability barrier exists for $N a_{\text {scat }} / a_{\mathrm{HO}}>1.9 \pm 0.1$. The dependence of the energy barrier on $N a_{\text {scat }} / a_{\mathrm{HO}}$ is shown in Fig. 5. In the inset of Fig. 5, the variational energy of the trial function $\Psi$ is shown as a function of $\left|C_{0}\right|^{2}$ for an interaction strength below (a) and above (b) the threshold value for metastable vortices. The calculations were performed for a configuration so that $A_{0}=5 \hbar \Omega, W_{0}^{2}$ $=20 a_{\mathrm{HO}}$. Note that the method of Ref. [27] followed here does not describe the decay of vortices mediated by the excitations, for example by nucleation of vortex loops. However, for a two-dimensional system punctured by an offresonance laser beam, Benakli et al. [27] could show that excitation-mediated decay of vortices only becomes appreciable for vortices in higher angular momentum states. Furthermore, for a purely one-dimensional toroidal system, Kagan et al. [29] showed that for a weakly interacting system at low temperature, the relaxation time for the decay of the superfluid persistent current due to phonons is strongly suppressed.

Both the method of Fetter [7] and the method of Benakli et al. [27] give qualitatively the same result, namely that above a critical strength of interaction, expressed by $N a_{\text {scat }} / a_{\mathrm{HO}}$, the vortex state of the LG condensate is stabilized by a metastability barrier. Quantitative differences remain between the two methods, which is to be expected since they describe different possible mechanisms of vortex decay. The general conclusion - the stabilization of the vortex state due to the toroidal geometry - is further supported by a calculation for a vortex condensate in a Mexican hat potential [30]. The metastability barrier calculated by the method of Ref. [7] is smaller than the barrier calculated used the method of Ref. [27] for $N a_{\text {scat }} / a_{\mathrm{HO}} \gtrsim 3.5$, which indicates that the latter method [27] overestimates the real metastability barrier.

\section{INTERFERENCE AND DETECTION OF VORTICITY}

Phase coherence, a property necessary for the existence of vortices, was demonstrated experimentally using interference experiments [31]. Two parabolically trapped condensates, displaced by a given distance, are allowed to expand freely. In the region where the two expanding condensates overlap, an interference pattern is observed. This type of experiment has also been proposed to observe vorticity in parabolically trapped condensates $[17,18]$ : the presence of a vortex leads to an observable edge dislocation in the pattern of otherwise parallel interference fringes.

Consider a parabolically trapped condensate positioned on the symmetry axis of the LG condensate. The LG condensate forms a cylindrical shell surrounding the parabolically trapped condensate in the center. As the trapping potentials are switched off, both condensates will expand and exhibit an interference pattern in the region of overlap. This interference pattern is the subject of the present section.

To find the function representing the order parameter at a given time $t$ after the start of the free expansion, the original function $\psi_{v}(\rho, \theta, z ; t=0)$ with variational parameters $\sigma, \zeta, \rho_{0}$ is expanded in free particle eigenfunctions. These eigenfunctions acquire a phase factor as time elapses, so that $\psi_{v}$ at a time $t$ is given by the resummed expansion with the "time evolved" eigenfunctions. More explicitly, for the LG condensate with a vortex, first the coefficients of the plane-wave expansion are evaluated:

$$
\begin{aligned}
& \psi_{v}(\rho, \theta, z ; t=0)=\int c(\mathbf{k}) \frac{e^{i \mathbf{k} \cdot \mathbf{r}}}{(2 \pi)^{3 / 2}} d \mathbf{k} \\
& c\left(k_{\rho}, \phi, k_{z}\right)= \int \psi_{v}(\rho, \theta, z) \\
& \times \frac{\exp \left\{-i k_{\rho} \rho \cos (\theta-\phi)-i k_{z} z\right\}}{(2 \pi)^{3 / 2}} d \mathbf{k} \\
&= \frac{\exp \left\{-k_{z}^{2} / 2 \zeta\right\}}{\sqrt{2 \pi \zeta}} \times e^{i(\phi+\pi / 2)} \int_{0}^{\infty} d \rho \rho^{2} J_{1}\left(k_{\rho} \rho\right) \\
& \times \exp \left\{-\sigma\left(\rho-\rho_{0}\right)^{2} / 2\right\} .
\end{aligned}
$$

In this expression, $J_{1}(x)$ is the Bessel function of first order of the first kind and the wave number $\mathbf{k}$ is expressed in cylindrical coordinates $\left\{k_{\rho}, \phi, k_{z}\right\}$. As time elapses, the freeparticle eigenfunctions acquire a phase factor 

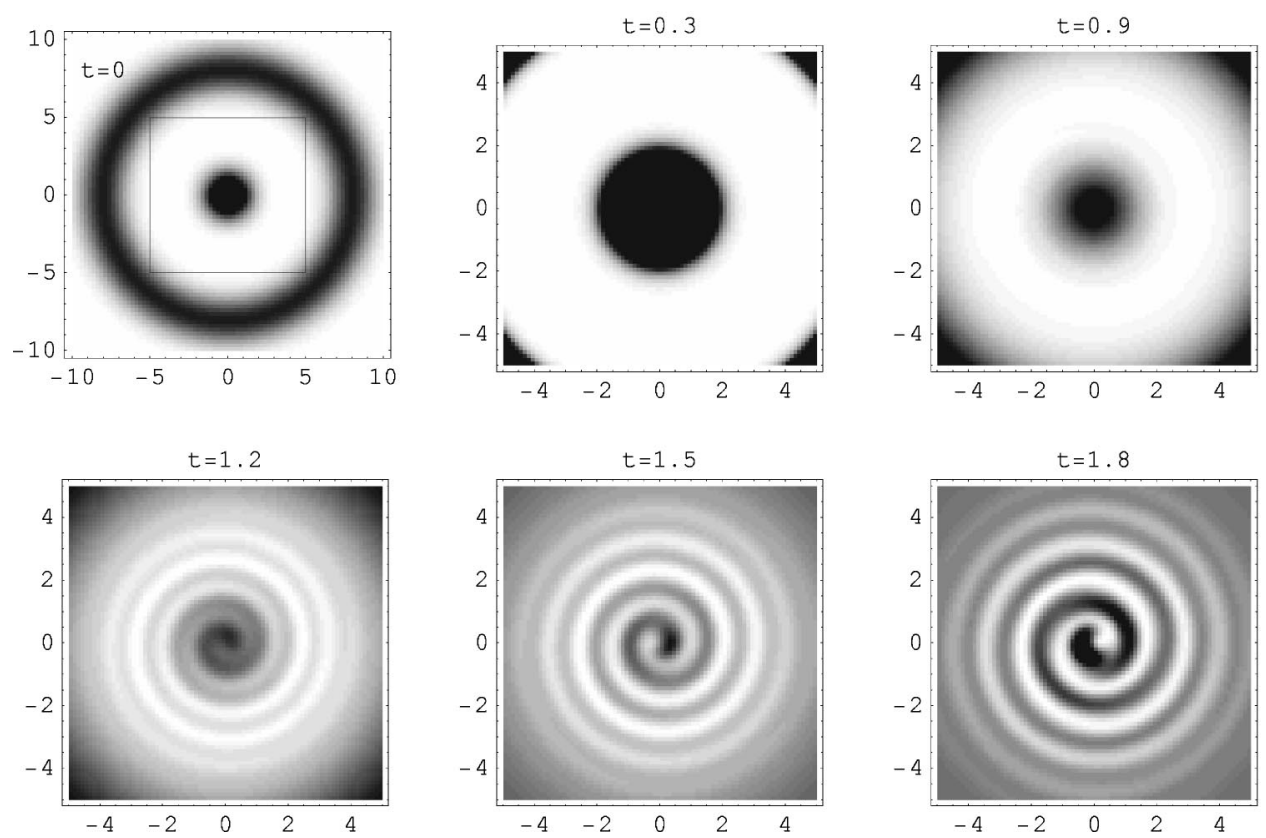

FIG. 6. Several time frames in the evolution of freely expanding, overlapping condensates are shown. The gray scale represents the density in a cross section orthogonal to the symmetry axis of the trap (the direction of propagation of the Laguerre-Gaussian laser beam), with black being the maximum density and white the minimum density. The initial situation is depicted in the top left panel: a parabolically trapped condensate in the center is surrounded by a cylindrical shaped Laguerre-Gaussian condensate containing a vortex. The time evolution of the density in the region within the rectangle in the top left panel is shown in more detail in the subsequent panels. When the confinement of both condensates is switched off, they expand and overlap - the density in the gap between both condensates increases — and a spiral interference pattern is formed. Units are chosen so that lengths are expressed in $a_{\mathrm{HO}}=\sqrt{\hbar /(m \Omega)}$ and the time is in units of $1 / \Omega$.

$\exp \left\{i \hbar k^{2} t /(2 m)\right\}$. The function at time $t$ after the start of the free expansion is found by resumming the eigenfunctions at time $t$ :

$$
\begin{aligned}
\psi_{v}(\rho, \theta, z ; t)= & \int c(\mathbf{k}) \frac{e^{i \mathbf{k} \cdot \mathbf{r}+i \hbar k^{2} t /(2 m)}}{(2 \pi)^{3 / 2}} d \mathbf{k} \\
= & \sqrt{\frac{1}{1+i \zeta t} \exp \left\{-\frac{\zeta z}{2(1+i \zeta t)}\right\}} \\
& \times e^{i \theta \int_{0}^{\infty} d k_{\rho}\left(\rho^{\prime}\right)^{2} \frac{-J_{1}\left(\rho^{\prime} \rho / t\right)}{t}} \\
& \times e^{-\sigma\left(\rho^{\prime}-\rho_{0}\right)^{2} / 2} \exp \left\{i \frac{\rho^{2}+\left(\rho^{\prime}\right)^{2}}{2 t}\right\} .
\end{aligned}
$$

The time evolution for the free expansion of a condensate prepared in a parabolic confinement is derived analogously [17]. The total measured density generated by the two condensates is then given by $\left|\psi_{v}(\rho, \theta, z ; t)+\psi(\rho, \theta, z ; t)\right|^{2}$ (remember that each condensate function is normalized to the number of particles in the given condensate). Several time frames of the resulting evolution of the density of the expanding condensates are shown in Fig. 6. The frames in Fig. 6 show a cross section of the density along the $x y$ plane.

Immediately after the traps are switched off, the density is that of a cylindrical, Laguerre-Gaussian condensate with a parabolically trapped condensate in the middle. As time goes by, both condensates expand: the parabolically trapped condensate expands radially, and the cylindrical shell of the LG condensate broadens. As the expanding condensates start to overlap, the fringe pattern appears. If the LG condensate does not contain a quantum of superfluid circulation (i.e., no vortex), the interference pattern consists of a series of concentric circles with linearly increasing radius. If, however, the LG condensate does contain a vortex, the interference pattern is an Archimedean spiral. At higher vorticity, the number of arms in the Archimedean spiral equals the number of vortex quanta in the LG condensate.

Figure 6 shows that, as a function of time, the spiral interference pattern rotates around the cylindrical symmetry axis of the trapped condensates, with a frequency of the order of the frequencies characterizing the parabolic approximation to the trapping potentials. Furthermore, as can be seen from Fig. 6, the distance between two successive windings of the spiral increases as time increases. Hence, to detect the spiral interference pattern, the density has to be measured on a time scale shorter than that given by the inverse of the characteristic trapping frequencies.

\section{CONCLUSIONS}

In conclusion, we have studied the properties of vortices in a condensate in an optical trap generated by a laser beam in a Laguerre-Gaussian mode and we proposed a method to detect these vortices. The energy of the vortex state was calculated variationally in a mean-field framework, and a threshold interaction strength was found beyond which there exists a metastability barrier stabilizing the LG vortex state 
against a transition to a nonvortex state. Both the hydrodynamic instability [7] and the uniform transition to a nonvortex state [27] were considered in the investigation of the vortex stability. Below the critical interaction strength, the metastability barrier vanishes, irrespective of the fact that the present confined geometry allows for the vortex core to be positioned in a region where the condensate is nonzero. The vanishing of the metastability barrier can be understood intuitively, since for the noninteracting Bose gas the critical velocity would vanish and superfluidity is destroyed.

The time evolution under free expansion of a LG condensate was derived and subsequently used to calculate the interference pattern that arises from the overlap of an expanding LG condensate with an expanding parabolically trapped condensate in its center. When superfluid circulation is present, the interference pattern consists of an Archimedean spiral, clearly distinct from the series of concentric cylindrical fringes that arise in the interference pattern when no vorticity is present. The observation of such a spiral interference pattern would constitute clear evidence for vorticity in spinpolarized Bose-Einstein condensates.

\section{ACKNOWLEDGMENTS}

We thank F. Brosens and L. F. Lemmens for intensive discussions. Also discussions with T. Kuga are gratefully acknowledged. This work was performed within the framework of the FWO Project Nos. 1.5.545.98, G.0287.95, 9.0193.97, G.0071.98, and WO.073.94N (Wetenschappelijke Onderzoeksgemeenschap, Scientific Research Community of the FWO on "Low Dimensional Systems"), the "Interuniversitaire Attractiepolen - Belgische Staat, Diensten van de Eerste Minister - Wetenschappelijke, Technische en Culturele Aangelegenheden," and in the framework of the GOA BOF UA 2000 projects of the Universiteit Antwerpen. One of the authors (J.T., aspirant bij het Fonds voor Wetenschappelijk Onderzoek - Vlaanderen) acknowledges the FWO for financial support.
[1] K. Huang, in Bose-Einstein Condensation, edited by A. Griffin, D. W. Snoke, and S. Stringari (Cambridge University Press, Cambridge, 1995), pp. 31-50.

[2] D. R. Tilley and J. Tilley, Superfluidity and Superconductivity (IOP Publishing, Bristol, 1990).

[3] M. H. Anderson, J. R. Ensher, M. R. Matthews, C. E. Wieman, and E. A. Cornell, Science 269, 198 (1995); C. C. Bradley, C. A. Sackett, J. J. Tollett, and R. G. Hulet, Phys. Rev. Lett. 75, 1687 (1995); K. B. Davis, M. O. Mewes, M. R. Andrews, N. J. Van Druten, D. S. Durfee, D. M. Kurn, and W. Ketterle, ibid. 75, 3969 (1995); D. G. Fried, T. C. Killian, L. Willmann, D. Landhuis, S. C. Moss, D. Kleppner, and T. J. Greytak, ibid. 81, 3811 (1998).

[4] W. Ketterle, D. S. Durfee, and D. Stamper-Kurn, in BoseEinstein Condensation in Atomic Gases, edited by M. Inguscio, S. Stringari, and C. Wieman (IOS Press, Amsterdam, 1999).

[5] F. Dalfovo and S. Stringari, Phys. Rev. A 53, 2477 (1996).

[6] D. S. Rokhsar, Phys. Rev. Lett. 79, 2164 (1997); A. A. Svidzinsky and A. L. Fetter, ibid. 84, 5919 (2000); D. A. Butts and D. S. Rokhsar, Nature (London) 397, 327 (1999).

[7] A. L. Fetter, J. Low Temp. Phys. 113, 198 (1998), e-print cond-mat/9808070; see also A. A. Svidzinsky and A. L. Fetter, Phys. Rev. A 62, 063617 (2000).

[8] B. Jackson, J. F. McCann, and C. S. Adams, Phys. Rev. Lett. 80, 3903 (1998).

[9] T. Isoshima and K. Machida, J. Phys. Soc. Jpn. 68, 487 (1999); T. Isoshima, K. Machida, and T. Ohmi, e-print cond-mat/0011421.

[10] L. Dobrek, M. Gajda, M. Lewenstein, K. Sengstock, G. Birkl, and W. Ertmer, Phys. Rev. A 60, R3381 (1999).

[11] K.-P. Marzlin, W. Zhang, and E. M. Wright, Phys. Rev. Lett. 79, 4728 (1997); K.-P. Marzlin and W. Zhang, Phys. Rev. A 57, 3801 (1998); B. M. Caradoc-Davies, R. J. Ballagh, and K. Burnett, Phys. Rev. Lett. 83, 895 (1999), e-print cond-mat/9902092.
[12] K. W. Madison, F. Chevy, W. Wohlleben, and J. Dalibard, Phys. Rev. Lett. 84, 806 (2000).

[13] M. R. Matthews, B. P. Anderson, P. C. Haljan, D S. Hall, C. E. Wieman, and E. A. Cornell, Phys. Rev. Lett. 83, 2498 (1999).

[14] C. J. Myatt, E. A. Burt, R. W. Ghrist, E. A. Cornell, and C. E. Wieman, Phys. Rev. Lett. 78, 586 (1997).

[15] S. A. Kennedy, J. T. Farrar, and E. R. I. Abraham, APS Centennial Meeting session NP01.25 (unpublished).

[16] T. Kuga, Y. Torii, N. Shiokawa, T. Hirano, Y. Shimizu, and H. Sasada, Phys. Rev. Lett. 78, 4713 (1997).

[17] J. Tempere and J. T. Devreese, Solid State Commun. 108, 993 (1998).

[18] E. L. Bolda and D. F. Walls, Phys. Rev. Lett. 81, 5477 (1998).

[19] D. M. Stamper-Kurn, M. R. Andrews, A. P. Chikkatur, S. Inouye, H.-J. Miesner, J. Stenger, and W. Ketterle, Phys. Rev. Lett. 80, 2027 (1998).

[20] B. E. A. Saleh and M. C. Teich, Fundamentals of Photonics (Wiley, New York, 1991).

[21] E. M. Wright, J. Arlt, and K. Dholakia, Phys. Rev. A 63, 013608 (2001); e-print cond-mat/0008479.

[22] S. Burger, K. Bongs, S. Dettmer, W. Ertmer, K. Sengstock, A. Sanpera, G. V. Shlyapnikov, and M. Lewenstein, Phys. Rev. Lett. 83, 5198 (1999).

[23] J. Denschlag, J. E. Simsarian, D. L. Feder, C. W. Clark, L. A. Collins, J. Cubizolles, L. Deng, E. W. Hagley, K. Helmerson, W. P. Reinhardt, S. L. Rolston, B. I. Schneider, and W. D. Phillips, Science 287, 97 (2000).

[24] J. Javanainen, S. M. Paik, and S. M. Yoo, Phys. Rev. A 58, 580 (1998); see also D.S. Rokhsar, e-print cond-mat/9709212.

[25] L. P. Pitaevskii, Zh. Éksp. Teor. Fiz. 40, 646 (1961).

[26] J. Tempere, F. Brosens, J. T. Devreese, and L. F. Lemmens, Solid State Commun. 107, 51 (1998); A. Parola, L. Salasnich, and L. Reatto, Phys. Rev. A 57, R3180 (1998); J. Tempere, F. Brosens, L. F. Lemmens, and J. T. Devreese, ibid. 61, 043605 (2000). 
[27] M. Benakli, S. Raghavan, A. Smerzi, S. Fantoni, and S. R. Shenoy, Europhys. Lett. 46, 275 (1999).

[28] B. P. Anderson, P. C. Haljan, C. E. Wieman, and E. A. Cornell, Phys. Rev. Lett. 85, 2857 (2000).

[29] Yu. Kagan, N. V. Prokofev, and B. V. Svistunov, Phys. Rev. A
61, 045601 (2000).

[30] L. Salasnich, A. Parola, and L. Reatto, Phys. Rev. A 59, 2990 (1999).

[31] M. R. Andrews, C. G. Townsend, H. J. Miesner, D. S. Durfee, D. M. Kurn, and W. Ketterle, Science 275, 637 (1997). 